\section{Diferencias entre los ventiladores mecánicos y los de las máquinas de anestesia en pacientes que sufren COVID-19}

\author{
Differences among the mechanical ventilators and those of \\ anesthesia machine, in patients suffering COVID-19
}

Prof. Dra. Cs. Idoris Cordero-Escobar,* Prof. Dra. Rosa María Abad-Hernández, ${ }^{\ddagger}$ Prof. Dr. Manuel Rivero-Moreno, ${ }^{\ddagger}$ Prof. Dra. C Katia Velázquez-González, ${ }^{\S}$ Dr. Reinier Hernández-González

\begin{abstract}
RESUMEN. Introducción: Los ventiladores de las MA constituyen un respaldo obvio de primera línea durante la pandemia COVID-19 cuando no hay suficientes ventiladores en las UCI para satisfacer las necesidades de atención del paciente. Objetivo: Actualizar a los médicos, que no son anestesiólogos, sobre las diferencias entre los ventiladores mecánicos y los de la máquina de anestesia, de manera que puedan ayudar a ventilar a los pacientes que sufren COVID-19. Desarrollo: Se revisaron los protocolos de actuación de varias Sociedades de Anestesiología y Reanimación, así como de Medicina Intensiva, de varios países, fundamentalmente de España, Chile, México y el Protocolo emitido por la CLASA sobre las consideraciones de cada uno de ellos para enfrentar, en caso de ser necesario, la ventilación mecánica con máquinas de anestesia en pacientes con COVID-19. Conclusiones: Las máquinas de anestesia modernas y los ventiladores mecánicos tienen similitudes y diferencias; pero ante esta situación, constituye un verdadero apoyo para que en lugares donde no existen suficientes ventiladores o no alcancen, la máquina de anestesia sea la sustituta de elección para ventilar a los pacientes con COVID-19.
\end{abstract}

ABSTRACT. Introduction: Anesthesia machines ventilators provide an obvious front-line backup during the COVID-19 pandemic when there are not enough ICU ventilators to meet patient care needs. Objective: To update the doctors which are not anesthesiologist, about the differences among the mechanical ventilators and those of anesthesia machine, in order to make them able, to help with the mechanical ventilation of patients suffering COVID-19. Development: The protocols of action of several Societies of Anesthesiology and Reanimation, as well as of Intensive Medicine, of several countries, mainly Spanish, Chilean, and Mexican, were reviewed on the considerations of each one to face in case mechanical ventilation is necessary, with anesthesia machines in patients with COVID-19. Conclusions: Modern anesthesia machines and mechanical ventilators have differences; but in this situation, it is a real support so that in places where there are not enough ventilators or they do not reach, the anesthesia machine is the substitute of choice to ventilate patients with COVID-19.

\section{INTRODUCCIÓN}

L as máquinas de anestesia (MA), en un principio, no fueron creadas para ventilar de forma $\_$prolongada a los pacientes. Toleran largos períodos de trabajo, pero no era el objetivo para la cual fue concebida.

Los ventiladores de las MA constituyen un respaldo obvio de primera línea durante la pandemia COVID-19 cuando no hay suficientes ventiladores en la Unidad de Cuidados Intensivos (UCI) para satisfacer las necesidades de atención al paciente. Los recursos y las limitaciones locales influirán en la mejor manera de aplicar esta solución ${ }^{(1)}$. Sin embargo, el curso de esta pandemia y la escases de recursos existentes en todo el mundo hizo que la agencia regulatoria de los Estados Unidos aprobara, temporalmente, el uso de dichas máquinas como ventiladores en las Unidades de Cuidados Intensivos para tratar de paliar su déficit en pacientes con falla ventilatoria por COVID-19(2).
Anestesiología

Octubre-Diciembre 2020

Vol. 43. No. 4. pp 284-287



Palabras clave:

Máquinas de anestesia, ventiladores mecánicos, insuficiencia respiratoria, COVID-19.

Keywords:

Anesthesia machines, mechanical ventilators, respiratory failure, COVID-19.

* Especialista en Anestesiología y Reanimación. Profesora e Investigadora Titular. MD, PhD. Servicio de Anestesiología y Reanimación. Hospital Hermanos Ameijeiras. La Habana, Cuba.

‡ Especialista en Anestesiología y Reanimación. Profesor Auxiliar. Servicio de Anestesiología y Reanimación. Hospital Hermanos Ameijeiras. La Habana, Cuba.

$\S$ Especialista en Anestesiología y Reanimación. MD, PhD Profesora Auxiliar. Jefa del Servicio de Anestesiología y Reanimación. Hospital Hermanos Ameijeiras. La Habana, Cuba.

" Especialista en Anestesiología y Reanimación. Profesor Asistente. Servicio de Anestesiología y Reanimación. Hospital Hermanos Ameijeiras. La Habana, Cuba.

Solicitud de sobretiros: Dra. Idoris Cordero Escobar Servicio de Anestesiología y Reanimación. 5to piso, Hospital Hermanos Ameijeiras, San Lázaro Núm. 701 entre Belascoain y Marques González, Centro Habana. La Habana, Cuba, 10300. E-mail: ice@infomed.sld.cu

Recibido para publicación: 20-04-2020

Aceptado para publicación: 08-07-2020 
Existen países desarrollados que ya han tomado esta iniciativa. Un ejemplo de ellos son China, España, Italia y Estados Unidos, que incluso han publicado diferentes protocolos al respecto a disposición de los médicos ${ }^{(3-5)}$.

Para su utilización es necesario la comprensión de las capacidades de las máquinas disponibles, las diferencias entre las MA y los ventiladores de la UCI y cómo establecer controles para imitar estrategias de ventilación en esta eventualidad.

La mayor experiencia acerca de COVID-19 procede del brote de China ${ }^{(6)}$. En este contexto, $80 \%$ de los pacientes confirmados tuvieron sintomatología leve a moderada, $13.8 \%$ tuvieron un curso clínico grave (disnea, taquipnea $\geq 30 / \mathrm{min}$, saturación $\mathrm{O}_{2} \leq 93 \%, \mathrm{PaO}_{2} / \mathrm{FiO}_{2} \leq 300$, y/o infiltrados pulmonares de $\geq 50 \%$ de los campos radiológicos y entre 24 y $48 \%$ presentaron un curso crítico de insuficiencia respiratoria, choque séptico y/o falla multiorgánica ${ }^{(6)}$.

Con los datos acumulados hasta el momento, en la Unión Europea y el Reino Unido, 30\% de los casos confirmados con COVID-19 requirieron ingreso y $4 \%$ se consideraban en estado crítico, definido como la condición que pone en peligro la vida del paciente, la necesidad de ventilación mecánica y de ser atendidos en la $\mathrm{UCI}^{(6)}$.

En España, entre los primeros 18,609 casos con información completa, 43\% requirieron ingreso hospitalario y 3.9\% ingreso en $\mathrm{UCI}^{(6)}$.

Según las experiencias en Wuhan, China, 15\% del total de pacientes infectados por esta enfermedad fueron hospitalizados y entre $5-10 \%$ fueron ventilados ${ }^{(1,2,5)}$. La Organización Mundial de la Salud ${ }^{(4)}$ publicó que $5 \%$ de pacientes infectados requirieron ingreso en la UCI y ventilación mecánica.

Nuestro objetivo constituye en actualizar a los médicos que no son anestesiólogos sobre las diferencias entre los ventiladores mecánicos y los de la máquina de anestesia de manera que puedan ayudar a ventilar a los pacientes que sufren COVID-19.

\section{DESARROLLO}

En Cuba existe un número de MA con prestaciones para ventilar pulmones enfermos. Dentro de ellas se encuentran algunos modelos de la Dräger (Fabius GS, Fabius PLUS, Fabius PRIMUS), Mindray WATO EX 35 y MAQUET Flow-i, cuyas casas comerciales emitieron documentos de orientación sobre este uso en etiquetas visibles. Estos documentos contienen recomendaciones útiles para el uso a largo plazo de estas máquinas como ventiladores en UCI.

Para la ejecución de este artículo se ha tomado como apoyo a las guías, sugerencias y recomendaciones de los diferentes países $^{(7-12)}$, fundamentalmente las recomendaciones del Comité Científico Sociedad Chilena de Medicina Intensiva y Anestesiología ${ }^{(3)}$, con indicación terapéutica de ventilación mecánica que eventualmente son conectados a las MA para la conducta ventilatoria en pacientes con COVID-19, y el de la Confederación Latinoamericana de Sociedades de Anestesiología (CLASA).

Por tal razón, se decidió identificar las diferencias entre ambos equipos de manera que el personal de perfil no anestesiológico pueda colaborar en caso de tener que utilizarlas en el curso de esta pandemia:

- Las MA poseen circuitos circulares que pueden funcionar con reinhalación total (flujos bajos), con reinhalación parcial (flujos medios) y sin reinhalación (flujos altos), mientras que los ventiladores mecánicos convencionales de la UCI siempre funcionan con circuitos circulares sin reinhalación.

- Se prefieren ventiladores anestésicos con compensación de compliance y entrega de volumen tidal no afectado por el flujo de gas fresco, pues proporcionan una entrega de volumen tidal más consistente y una supervisión más precisa.

- La válvula de presión AVL no se debe utilizar. Ésta regula la presión en ventilación espontánea y permite que el paciente ventile espontáneamente.

- En las MA, dado que pueden emplear flujos bajos, medios $\mathrm{y}$ altos, vienen dotadas de medidores de flujo, mientras que los ventiladores de la UCI siempre funcionan con flujos altos y no están dotados de medidores de flujo.

- Las MA más capaces se deben utilizar primero y es preferible utilizar el mismo modelo en lugar de introducir diferentes equipos en la UCI. Se debe priorizar aquéllas unidades que desarrollen, por lo menos, estas modalidades de ventilación: ventilación controlada por volumen y por presión, ventilación intermitente sincronizada con presión asistida y ventilación asistida por presión.

La instalación de la máquina requerirá:

- Retirar los monitores y computadoras que se montan en la MA antes de su uso en una UCI.

- Retirar las conexiones de oxígeno presurizado y aire, ya sea a la tubería del hospital o grandes cilindros G o H. Se debe contar con oxígeno de reserva y cilindros de aire.

- Eliminar todos los vaporizadores.

- Eliminar los cilindros de óxido nitroso y mangueras de tubería.

- Si es posible modificar los parámetros del ventilador predeterminado y los ajustes de alarma (por ejemplo, nivel PEEP, volumen de alarma, ventilación de minutos y alarmas de presión de las vías respiratorias, entre otros) para que coincidan con los de los ventiladores UCI.

- Ajustar el sistema de eliminación (las salidas de aspiración están disponibles en la UCI, pero no se pueden conectar a la conexión WAGD de la máquina debido a la incompatibilidad del conector). 
- Cambiar el gas de transmisión si el ventilador de anestesia contiene un fuelle. El oxígeno al 100\% es el gas de transmisión estándar para un ventilador tipo fuelle y se consume aproximadamente en la ventilación minuto (que es significativamente mayor que el consumo de oxígeno de gas fresco). Si los suministros de oxígeno son limitados o conservados, se pueden hacer modificaciones en algunas máquinas de anestesia para utilizar aire comprimido como el gas de transmisión. Estas modificaciones pueden ser realizadas en menos de una hora por un ingeniero clínico entrenado siguiendo las instrucciones del fabricante.

- Las capacidades de ventilación de la mayoría de las MA, incluso los que tienen limitados los modos de ventilación y la ausencia de PS, deben ser suficientes como intervención para salvar la vida de la mayoría de los pacientes.

- La monitorización respiratoria es importante. Ésta incluye la monitorización de presión y flujo con alarmas. La espirometría en tiempo real (flujo-volumen y curva presión-volumen), es muy útil cuando se atiende a pacientes con insuficiencia respiratoria y para diagnosticar fugas alrededor del tubo endotraqueal y una mayor resistencia a través de la vía respiratoria con filtros intercambiadores de calor y humedad (HMEF).

- La utilización de oxígeno puede ser un factor a la hora de seleccionar los ventiladores de la MA y gestionar los modos de ventilación. En general, los ventiladores neumáticos consumen más oxígeno que los eléctricos; pero se describirán modificaciones para conservar el oxígeno con todos los diseños de ventiladores.

- El gas impulsor no se puede cambiar a aire en las MA Mindray.

- Un circuito respiratorio con bolsa de depósito debe tener filtros virales para proteger la máquina de la contaminación interna. Se debe colocar un filtro de intercambio de calor y humedad (HMEF) en la conexión del tubo endotraqueal al circuito respiratorio (Y) y un segundo que sólo realice intercambio de calor y humedad (HME) en la manguera espiratoria donde se conecta a la MA.

- Los filtros de recambio y los circuitos respiratorios deben estar fácilmente disponibles.

- Un resucitador manual de respaldo (bolsa-válvula-máscara) con filtro en el puerto de expiración que está disponible en todo momento para la ventilación de respaldo.

- Se debe tener en cuenta que, si en algunos modos de ventilación fallara en el ventilador de anestesia, la ventilación manual de la MA puede funcionar correctamente.
- Monitor de gases respiratorios para oxígeno inspirado y dióxido de carbono inspirado/exhalado, interno o externo a la máquina.

- La mayoría de las MA modernas tienen procedimientos de prueba de inicio para ser realizados antes de su uso, estos deben ser repetidos cada 24 horas para asegurar el funcionamiento adecuado.

- El paciente no puede ser ventilado con la MA durante la prueba de inicio, incluso en modo manual, por lo que se necesitan medios alternativos de ventilación durante este tiempo. Aunque no se considera ideal, la orientación de los fabricantes durante esta crisis permite un intervalo de prueba de inicio de hasta 72 horas.

- Consulte la lista de comprobación de pruebas de inicio para obtener una guía paso a paso.

Otras consideraciones a tener en cuenta son:

- Que la MA entregue una concentración de oxígeno inspirada deseada. Si no dispone de sensor de oxígeno se debe adjuntar una tabla para conocer la $\mathrm{FiO}_{2}$ de acuerdo con la proporción de flujo de oxígeno y aire.

- Los circuitos recomendados son sin reinhalación, por lo tanto, el flujo total de gases debe estar en $20 \%$ por encima del volumen minuto.

- Como los circuitos empleados son sin reinhalación la cal sodada no debe agotarse, pero aun así se debe observar el indicador de agotamiento y si existe reinhalación de $\mathrm{CO}_{2}$.

- No se recomienda, por los que tienen experiencia en la utilización de la MA para ventilar pacientes en la UCI, utilizar gases anestésicos para su sedación, por el costo de los mismos y por la contaminación ambiental. Se recomienda las mismas técnicas de sedación que se emplean en las UCI.

- Se recomienda que siempre que se empleen las máquinas de anestesia para ventilar a estos pacientes debe existir un anestesiólogo para supervisar la actividad.

Se concluye que las máquinas de anestesia modernas y los ventiladores mecánicos tienen diferencias; pero ante esta situación, constituye un verdadero apoyo para que en lugares donde no existen suficientes ventiladores o no alcancen, la máquina de anestesia sea la sustituta de elección para ventilar a los pacientes con COVID-19. 


\section{REFERENCIAS}

1. FDA. Regulaciones sobre la ventilación mecánica con máquinas de anestesia. [En línea]. (Consultado: 25 abril 2020). URL: https://www. fda.gov/medical-devices/letters-health-care-providers/ventilator-supplymitigation-strategies-letter-health-care-providers.

2. American Society of Anesthesiologists ASA y la Anesthesia Patient Safety Foundation APSF. Guía APSF/ASA sobre el uso de las máquinas de anestesia como ventiladores de UCI Documento original en inglés: [En línea] (Consultado: 20 abril 2020). URL: https://www.asahq.org/inthe-spotlight/coronavirus-covid-19-information/purposing-anesthesiamachines-for-ventilators.

3. Comité Científico Sociedad Chilena de Medicina Intensiva, Sociedad Chilena de Anestesiología. Recomendaciones para el manejo de pacientes con COVID-19 con indicación terapéutica de ventilación mecánica que eventualmente son conectados a máquinas de anestesia. Resumen ejecutivo. Marzo 2020. [En línea] (Consultado 22 Marzo 2020). URL: www.colegiomedico.cl/wp-content/uploads/2020/04/ revchilanestv49n03.09.pdf

4. Confederación Latinoamericana de Sociedades de Anestesiología (CLASA). Protocolo COVID-19.

5. World Health Organization. Statement on the second meeting of the International Health. Regulations. Emergency Committee regarding the outbreak of novel coronavirus (2019-nCoV). 2020 [En línea] (Consultado 22 Marzo 2020). URL: https://www.who.int/newsroom/detail/30-012020-statement-on-the-second-meeting-of-theinternational-healthregulations-(2005)-emergency-committeeregarding-the-outbreakofnovel-coronavirus-(2019-ncov).
6. Herman JA, Urits I, Kaye AD. COVID-19: General anesthesia Precautions. J Clinical Anesth (2020). [En línea] (Consultado 26 Marzo 2020). URL https://doi.org/10.1016/j.jclinane.2020.109840.

7. Información científica-técnica. Enfermedad por coronavirus, COVID-19 Actualización 4 de abril 2020. (Consultado 15 marzo 2020). URL. Centro de Coordinación de Alertas y Emergencias Sanitarias. Ministerio de Salud España. Secretaría de Sanidad y Consumo. Dirección General de Salud Pública, Calidad y consumo. [En línea] (Consultado: 25 abril 2020) URL. https://www.mscbs.gob.es/profesionales/saludPublica/ccayes/ alertasActual/nCov-China/documentos/20200404_ITCoronavirus.pdf.

8. Aranda F, Aliste J, Altermatt F, Alvarez JP, Bernucci F, Bruhn A, et al. Recomendaciones para el manejo de pacientes con COVID-19 con indicación terapéutica de ventilación mecánica que eventualmente son conectados a máquinas de anestesia. Rev Chil Anest 2020;49:196-202.

9. Kamps BS, Hoffmann C. COVID reference esp | 2020.1. [En línea] (Consultado 26 Marzo 2020). URL: www.CovidReference.com.

10. Aranda F, Aliste J, Altermatt F, Alvarez JP, Bernucci F, Cabrera MC. Recomendaciones para el manejo de pacientes con COVId19 en el perioperatorio. Rev Chil Anest. 2020;49:196-202.

11. Wax RS, Christian MD. Practical recommendations for critical care and anesthesiology teams caring for novel coronavirus (2019-nCoV) patients. Can J Anesth. 2020. doi: https://doi.org/10.1007/s12630020-.

12. Peng PW, Ho PL, Hota SS. Outbreak of a new coronavirus: what anaesthetists should know. Br J Anesth. 2020;124(5):497-501. Feb; https://doi.org/10.1016/j. 